\title{
Article
}

\section{Abnormal empathy-like pro-social behaviour in the valproic acid model of autism spectrum disorder}

Fontes-Dutra, Mellanie, Della-Flora Nunes, Gustavo, Terra, Julio Santos, Souza-Nunes, Walquíria, Bauer-Negrini, Guilherme, Hirsch, Mauro Mozael, Green, Lily, Riesgo, Rudimar, Gottfried, Carmem and Bambini-Junior, Victorio

Available at http://clok.uclan.ac.uk/26042/

Fontes-Dutra, Mellanie, Della-Flora Nunes, Gustavo, Terra, Julio Santos, SouzaNunes, Walquíria, Bauer-Negrini, Guilherme, Hirsch, Mauro Mozael, Green, Lily, Riesgo, Rudimar, Gottfried, Carmem et al (2019) Abnormal empathy-like prosocial behaviour in the valproic acid model of autism spectrum disorder. Behavioural Brain Research, 364 . pp. 11-18. ISSN 0166-4328

It is advisable to refer to the publisher's version if you intend to cite from the work. http://dx.doi.org/10.1016/j.bbr.2019.01.034

For more information about UCLan's research in this area go to http://www.uclan.ac.uk/researchgroups/ and search for <name of research Group>.

For information about Research generally at UCLan please go to http://www.uclan.ac.uk/research/

All outputs in CLoK are protected by Intellectual Property Rights law, including Copyright law. Copyright, IPR and Moral Rights for the works on this site are retained by the individual authors and/or other copyright owners. Terms and conditions for use of this material are defined in the policies page. 


\section{Accepted Manuscript}

Title: Abnormal empathy-like pro-social behaviour in the valproic acid model of autism spectrum disorder

Authors: Mellanie Fontes-Dutra, Gustavo Della-Flora Nunes, Julio Santos Terra, Walquíria Souza-Nunes, Guilherme Bauer-Negrini, Mauro Mozael Hirsch, Lily Green, Rudimar Riesgo, Carmem Gottfried, Victorio Bambini-Junior

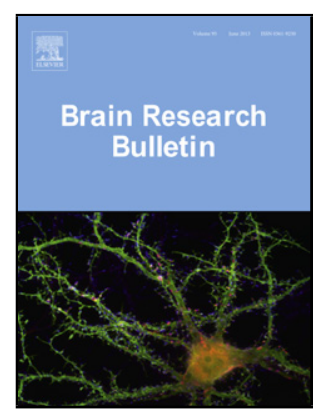

PII:

DOI:

Reference:

S0166-4328(18)31114-8 https://doi.org/10.1016/j.bbr.2019.01.034

To appear in: $\quad$ Behavioural Brain Research

Received date: $\quad 5$ August 2018

Revised date: $\quad 17$ December 2018

Accepted date: 18 January 2019

Please cite this article as: Fontes-Dutra M, Della-Flora Nunes G, Terra JS, Souza-Nunes W, Bauer-Negrini G, Hirsch MM, Green L, Riesgo R, Gottfried $\mathrm{C}$, Bambini-Junior V, Abnormal empathy-like pro-social behaviour in the valproic acid model of autism spectrum disorder, Behavioural Brain Research (2019), https://doi.org/10.1016/j.bbr.2019.01.034

This is a PDF file of an unedited manuscript that has been accepted for publication. As a service to our customers we are providing this early version of the manuscript. The manuscript will undergo copyediting, typesetting, and review of the resulting proof before it is published in its final form. Please note that during the production process errors may be discovered which could affect the content, and all legal disclaimers that apply to the journal pertain. 


\section{a. TITLE PAGE}

\section{Abnormal empathy-like pro-social behaviour in the valproic acid model of autism spectrum disorder}

Mellanie Fontes-Dutra*,1,2,3, Gustavo Della-Flora Nunes ${ }^{\star, 1,3,4}$, Julio Santos Terra ${ }^{1,2,3}$, Walquíria Souza-Nunes ${ }^{1,2,3}$, Guilherme Bauer-Negrini ${ }^{1,2,3}$, Mauro Mozael Hirsch ${ }^{1,2,3}$, Lily Green ${ }^{5}$, Rudimar Riesgo ${ }^{1,2,6}$, Carmem Gottfried ${ }^{1,2,3}$, Victorio Bambini-Junior ${ }^{\#, 1,3,5}$

* These authors contributed equally to this work

1 Translational Research Group in Autism Spectrum Disorders-GETTEA, Universidade Federal do Rio Grande do Sul (UFRGS), Ramiro Barcelos street, 2600, 90035-003 - Porto Alegre, RS, Brazil. Phone: +55 51 3308-5551 2 Department of Biochemistry, Universidade Federal do Rio Grande do Sul (UFRGS), Ramiro Barcelos street, 2600, 90035-003 - Porto Alegre, RS, Brazil. Phone: +55 51 3308-5539.

3 National Institute of Science and Technology on Neuroimmunomodulation (INCT-NIM). Brasil avenue, 4365, Manguinhos, 21045-900 - Rio de Janeiro, RJ, Brazil.

4 Department of Biochemistry, University at Buffalo, The State University of New York, 955 Main Street, Suite 4102 - NY, USA. Phone: +1 716 829-2727, Fax: +1 716 829-2725

5 School of Pharmacy and Biomedical Sciences, University of Central Lancashire, PR1 2HE, Lancashire, Preston, England. Phone: +44 01772891990

6 Child Neurology Unit, Hospital de Clínicas de Porto Alegre (HCPA), Ramiro Barcelos, 2350, 90035-903 - Porto Alegre, RS, Brazil. Phone: $+55513359-8000$

\# Correspondent author: victoriobambini@gmail.com 


\section{b. ABSTRACT}

Impairments in social behaviour are a defining feature of autism spectrum disorder (ASD). Individuals with ASD also usually present some difficulty to recognise or understand another person's feelings. Therefore, it is possible that altered empathy processing could hinder typical social interaction in ASD. Recently, robust paradigms confirmed that rodents show primordial forms of empathy-like behaviour. Therefore, in this work, we used one of these new protocols to test pro-social behaviour in the rat model of autism induced by Valproic Acid (VPA). We also evaluated possible beneficial effects of Resveratrol, since it can prevent social deficits in the VPA model. Rats were tested on their ability to open a restrainer to release a trapped conspecific. Exposure to VPA precludes the timely manifestation of this empathy-like behaviour, but does not affect its continuation after its first expression. We also found a significant correlation between average speed during the first day of test and becoming an Opener. Similarly, rats able to open the restrainer on the first day had an increased likelihood of repeating this behaviour in the later days of the testing programme. We did not find any protective effects of Resveratrol. Further investigation of empathy-like behaviour in the VPA model and in other models of autism could help to clarify the behavioural and neural processes underpinning the basic aspects of empathy alterations in autistic individuals.

Keynotes: Animal models, valproate, empathy, autism, pro-social behaviour.

\section{Highlights:}

* Individuals with ASD can present deficits in empathy-dependent features.

* Empathy is a complex social manifestation recently described in rodents.

*VPA-induced animal model of ASD shows abnormal empathy-like pro-social behaviour.

* Pre-treatment with RSV can prevent social deficits but not empathy-like behaviours.

* Early tracking analysis provide strong correlation with the test outcomes. 


\section{INTRODUCTION}

Autism spectrum disorder (ASD) is a complex neurodevelopmental disorder characterized by social communication impairments, restricted interests and repetitive behaviours [1]. In addition, individuals with ASD often present several associated comorbidities such as epilepsy, sleep disturbances, immune alterations and impairments in emotional and empathy-driven responses [18, 38]. The aetiology and molecular basis of ASD are still unknown. However, different genetic alterations and environmental risk factors can contribute to the development of ASD [19].

Valproic Acid (VPA), or 2-propylpentanoic acid, is a fatty acid with anticonvulsant [28] and mood stabilizing [8] properties and it is a relevant drug for the treatment of epilepsy, bipolar disorder and other neuropsychological disorder. Epidemiological studies have demonstrated a strong link between the prenatal exposure to VPA and the onset of ASD in the offspring [9, 37]. Considering this association, an animal model for the study of ASD induced by prenatal exposure to VPA was established [43, 46].

Accumulating evidence demonstrates that the VPA model presents face validity (strong phenomenological similarities and related pathophysiology), construct validity (the same aetiology; in this case, exposure to VPA) and predictive validity (same response to treatments aiming to prevent or reverse symptoms) [30]. Therefore, this model has the potential to elucidate targets for pharmacological treatment optimization, and for the prevention or reversal of ASD-like symptoms [48]. For instance, our group demonstrated that resveratrol (RSV), an antioxidant and anti-inflammatory molecule, prevents VPA-induced social impairments in a three-chambered test [3] and in a reciprocal social interaction test [20], and lead us to use this approach to elucidate the underlying pathways associated with ASD and social behaviour.

Empathy is a complex phenomenon that could be understood in humans as the ability to understand and share the internal states of others, while generating an emotional response more appropriate to someone else's situation than to one's own. Therefore, empathy is frequently demonstrated through caring and helping behaviour toward others [49]. Several studies have indicated that individuals with ASD have impaired empathy behaviour, as manifested by the reduced ability of ASD subjects to recognize facial features [44], lower scores in the empathy quotient self-report questionnaire [5] and difficulties in identifying/understanding what another individual is thinking or feeling [27].

Despite the need for higher cognitive processing for the manifestation of some aspects of empathy behaviour in humans, it has been increasingly accepted that other animals also present primordial forms of empathy [23, 39]. For example, many species show emotional contagion, the capacity to mimic someone else's emotions [13], and an ability that could be at the foundation of empathy behaviour. In addition, it has long been known that rats can engage 
in pro-social acts to help conspecifics in distress [10,41], which is a behaviour that was recently further investigated $[6,45]$.

In a landmark paper, Bartal et al. showed that rats intentionally opened a restraining door to release a trapped cage-mate, but did not open an empty restrainer or a restrainer containing a toy rat [6]. In addition, animals continued to manifest this helping behaviour even when the trapped rat was released in a different arena, supporting the hypothesis that social reward was not necessary to release the trapped rat. Interestingly, when rats had the option to open a restrainer containing chocolate or a restrainer with a cage-mate, they showed no preference and shared the chocolate. In a follow up study, the same group demonstrated that this behaviour is socially acquired since rats did not release trapped animals of an unfamiliar strain. However, when reared with animals of this different strain, rats helped strangers of the fostering strain but not rats of their own strain [7].

The development of these new robust paradigms can now allow the first investigation of pro-social behaviour in an animal model of autism. Therefore, we aimed to evaluate the expression of empathy-like behaviours in the VPA model of autism. In addition, given the preventive actions of RSV on the social impairments shown by VPA rats, we also studied the effects of RSV on empathy-like behaviour. 


\section{MATERIALS AND METHODS}

\subsection{Animals}

Adult Wistar rats (200-300g) from the local breeding colony (ICBS-Federal University of Rio Grande do Sul) were housed in plastic cages (four to five per cage), under a $12 \mathrm{~h}$ light/dark cycle, controlled temperature $(22 \pm 1 \circ \mathrm{C})$ and humidity at $40-60 \%$. Water and food ad libitum. Animals were mated overnight and if in the morning spermatozoa were found in vaginal secretion, this day was designated as the first day of pregnancy [2]. Pregnant rats were housed in pairs until one day before the birth. The offspring were weaned at 21 days old and housed separately by sex. Only the male rats were included in the behavioural experiment. The empathy-like behaviours were evaluated in 69 to 81 days old animals from at least three pregnant female Wistar rats per treatment group, with two animals per litter tested. Test animals were housed with their brothers, respecting the aforementioned housing conditions. The same is valid for animals utilized as "trapped" animals. The behavioural test was conducted in a specialized room. Animals were taken in their home cage to this room 30 min before testing and were immediately reconducted to their original room after each daily trial. All animal experiments were approved by the local ethics committee (CEUA \# 23884) and followed the regulations of the Brazilian national committee for the control of animal experimentation (CONCEA) and the NIH guidelines for care and use of laboratory animals.

\subsection{The Animal Model}

At E12.5 pregnant female rats received a single intraperitoneal injection of $600 \mathrm{mg} / \mathrm{kg}$ VPA (Acros Organics, NJ, USA) or physiological saline. VPA was previously dissolved in $0.9 \%$ saline with a concentration of $250 \mathrm{mg} / \mathrm{mL}$ [2].

Prenatal treatment with resveratrol (Fluxome, Stenløse, Denmark) was administered from E6.5 to E18.5. Resveratrol (RSV) was dissolved in 100\% DMSO (Merck, New Jersey, USA) for a concentration of $36 \mathrm{mg} / \mathrm{mL}$. Pregnant female rats received a daily subcutaneous injection of $3.6 \mathrm{mg} / \mathrm{kg}$ of RSV solution or correspondent volume of DMSO, as previously described [3]. Based on treatment received the pregnant rats were separated in 4 experimental groups: control (received only DMSO injections from E6.5 to E18.5); RSV (received only RSV injections from E6.5 to E18.5); VPA (received DMSO from E6.5 to E18.5 plus VPA injection at E12.5); RSV + VPA (received RSV from E6.5 to E18.5 plus VPA injection at $\mathrm{E} 12.5)$. Two male rats of each litter were subjected to the behavioural analysis, resulting in the following sample sizes: Control (16), RSV (10), VPA (14) and VPA+RSV (10).

\subsection{Restrainer Tube and Empathy-Like Behaviour}


We used a modified protocol based on Bartal et al. [6]. In short, the test consisted of the evaluation of the willingness of rats to open a restrainer where a conspecific was trapped. The apparatus was constructed in Plexiglas (25 x $8.75 \times 7.5 \mathrm{~cm}$; length, width, height) and had a customized door that only opened from the outside. A schematic figure of the restrainer is shown in Supplementary Figure 5. The restrainer was placed inside a black wooden box $(50 \mathrm{~cm} \times 50 \mathrm{~cm} \times 50 \mathrm{~cm})$, and was cleaned and disinfected with alcohol $(70 \%)$ after each trial. Room luminosity was set to 60 lux.

Before the test, animals were habituated in the apparatus according to the following schedule (not recorded in video):

Day 1: two animals from the same litter were handled by an investigator for 5 minutes and placed in the black box containing the acrylic tube (open and empty) for 60 min.

Day 2: two animals from the same litter were handled by an investigator for 10 minutes and placed in the black box containing the acrylic tube (open and empty) for 60 min.

Day 3: the animals were handled for 5 minutes and placed individually in the black boxes containing the acrylic tubes (open and empty) for $60 \mathrm{~min}$.

Before every procedure during the test days, animals were placed inside the test room for 30 minutes in order to get habituated to the new environment. The trials were completed over 12 days, with an hour for each trial on each day. All trial days were recorded using a full HD webcam (Logitech C920 pro full HD $1920 \times 1080$ pixels, with a maximum frame rate of $30 \mathrm{fps}-1080 \mathrm{p})$. The restrained animal was placed in the restraining tube and the door closed. The restraining tube was then placed in the centre of the wooden box. The free rat was placed next to the restraining tube and recording began. The animals restrained in the acrylic tube were 60 days old male Wistar rats that had not been previously submitted to any intervention and were smaller (10\% less body weight, at least) and unknown to the test animals. Trapped animals were also randomly rotated between each trial session, so that test animals had to release different trapped animals in different days of the test. This strategy was used in order to minimize the effect of the trapped animal on the behaviour of the test subjects.

If the door was not opened within the first 40 minutes of the trial, the door was lifted halfway in a $45^{\circ}$ angle, and a Plexiglas blocker was inserted to prevent door opening by the trapped rat. Any openings that occurred after this point were not included in analysis. Animals were not taught how to open the door or rewarded for that task in any moment. The opening occurrence and latency were registered and movements were tracked using ANYmaze 5.

Animals were defined as openers if they were able to open the restrainer for two consecutive days and to do so at least 3 times [7]. For example, if a rat opens the restrainer for four consecutive days or more, it is considered an opener (three sequences of 2 days: $1^{\text {st }}$ and $2^{\text {nd }}, 2^{\text {nd }}$ and $3^{\text {rd }} ; 3^{\text {rd }}$ and $4^{\text {th }}$ days). 
The acrylic tubes were set up to indicate an inner zone (where the free rat could be considered to be interested in the restrained rat), an outer perimeter zone (where the free rat could then be considered to not showing interest) and a door zone (where the free rat could be considered to be demonstrating active interest in the door of the restraining tube) (Figure 1). The zones were discriminated in ANYmaze 5 and the position of the head of the free rats was tracked, allowing the automated calculation of time spent and distance travelled in each zone. Speed values were calculated by dividing distance and time parameters for each individual. Group values were calculated as averages of the individuals. For each trial, tracking was terminated when the trapped animal was released. Therefore, test duration is not the same across individuals/days and most results are reported as percentages. When rats were inside the door or inner zone, we observed the presence of exploratory behaviours, such as sniffing, following alongside the restrainer cage, rearing and climbing in the restrainer cage. $\mathrm{A}$ representative video of the behavioural task is available in the supplemental material.

All the data was gathered and analysed attempting to minimize subjective bias. Original videos were renamed and had all identification erased before analysis in order to avoid the chance of bias. In addition, most parameters analysed were calculated in an automated way using the ANYmaze software.

\subsection{Statistical Analysis}

All behavioural parameters were analysed using Generalized Estimating Equations (GEE) and were adjusted by litter. The GEE is a flexible method based on general linear models, allowing the analyses of data with different distribution patterns. The GEE was utilized to allow for the evaluation of the effect of the many correlated variables in our analysis (VPA treatment, RSV treatment, opener outcome and their interactions). One of the main advantages of GEE is the reduced sample size required to detect a same size effect as compared to similar methods. The GEE was modelled using gamma distribution for all the following parameters: average speed, average speed in inner zone and in door zone, distance travelled, percentage in inner zone and in door zone head distance travelled, percentage in inner and in door zone head times, litter size and percentage of males and females per litter. For the comparison of littermates, we modelled the GEE using gamma distribution for all average speeds and percentages. We used Poisson distribution for quantitative analyses of number of openings, day of first opening and percentage of miscarriages in each group and Normal distribution for total distance travelled. The Wald test was used as inference test. For analyses of correlation with the opener outcome, we used a Binomial distribution. Post-hoc analyses were performed and the p-value was adjusted for multiple comparisons using Bonferroni correction. All the estimated means were described as mean \pm SEM and results were considered significant when $\mathrm{p}<0.05$. 


\section{RESULTS}

\subsection{Effects of prenatal exposure to VPA and RSV on rat pregnancy}

We first analysed the pregnancy outcomes for a larger cohort of animals that included the animals in this study (Control $n=24, R S V n=22$, VPA $n=23$, VPA $+R S V n=27$ ). VPA significantly increased the number of miscarriages (Control: $12.50 \pm 0.72$, RSV: $22.73 \pm 1.02$, VPA: 47.83 \pm 1.44, VPA+RSV: 44.44 $\pm 1.28, p=0.017$, Supplementary Figure $1 A)$. Miscarriages were defined as events in which females that had a vaginal plug detected did not give birth to a single pup. The percentage of miscarriages is almost identical to data previously reported [15]. Prenatal exposure to VPA or RSV had no effect on litter size (Supplementary Figure 1B). There was also no difference in the percentage of males/females in each litter (Supplementary Figures $1 \mathrm{C}$ and 1D). Pup mortality was a rare event and only happened in 2 litters of the VPA group.

\subsection{Prenatal exposure to VPA delayed the timely manifestation of empathy-like behaviour in Wistar rats}

Animals exposed to VPA demonstrated a delay in the day of first opening in relation to the Control and RSV groups (Control: $3.15 \pm 0.64$, RSV: $3.14 \pm 0.91$, VPA: $5.92 \pm 0.98$, VPA+RSV: $6.62 \pm 1.04, p=0.001$ ) and RSV treatment was incapable of preventing this alteration (Figure 2A). Consequently, animals of the VPA and VPA+RSV groups demonstrated a tendency for reduction in the total number of openings $(p=0.056)$, which was also not prevented by RSV treatment (Figure $2 \mathrm{~B}$ ). The Supplementary Figure $2 \mathrm{~A}$ illustrates each individual opening event. There was no difference in the percentage of animals of each group that matched the opener criteria (Supplementary Figure 2B). We also calculated the percentage of openings, defined as the percentage of trial sessions in which the test animals were able to release the trapped conspecific. The percentage of openings on each day is illustrated on Supplementary Figure 2C. When the data for the percentage of openings was arranged in groups of 4 days, animals exposed to VPA showed a reduced percentage of openings in days 1 to 4 (Control: $35.93 \pm 8.53$, RSV: $35 \pm 11.9$, VPA: $14.28 \pm 6.79$, VPA+RSV: $5 \pm 3.33 p=0.009$ ) (Figure $2 C$ ). Interestingly, once animals opened the restrainer for the first time, they continued to open it at the same rate, regardless of the experimental group (Figure 2D). Animals that were unable to open the restrainer at least once were excluded from this last analysis.

\subsection{Average speed increases in the day of first opening}

With the hope of gaining valuable insights on the behavioural patterns important for the expression of this empathy-like prosocial behaviour, we analysed the day of first opening 
of rats and compared this with the behaviour of littermates that did not open the restrainer on that day. We observed a significant increase in the average speed of animals that opened the restrainer when compared to their littermates (Con-O: 6.57 \pm 0.9 , Con-NO: $1.78 \pm 0.25$, RSV-O: 6.86 \pm 0.9 , RSV-NO: $1.9 \pm 0.49$, VPA-O: $5.35 \pm 0.33$, VPA-NO: 1.8 \pm 0.47 , VPA+RSV-O: $4.6 \pm 0.58$, VPA+RSV-NO: $2.22 \pm 0.70, p<0.001$, Figure $3 A$ ). Regions of the test box were also analysed separately and the resulting data pointed out to differences in the average speed when in the door zone (Con-O: 4.5 \pm 0.6 , Con-NO: $2.7 \pm 0,4$, RSV-O: 6.6 \pm 1.1 , RSV-NO: 2.0 $\pm 0,7$, VPA-O: 4.5 $\pm 1,0$, VPA-NO: $2.2 \pm 1.1$, VPA+RSV-O: $3.5 \pm 0.4$, VPA+RSV-NO: $2.8 \pm 0.7 ; p=0.004$, Figure 3B). Similar results were also found for the inner zone of the apparatus (Con-O: $5.88 \pm 0.8$, Con-NO: 2.65 \pm 0.42 , RSV-O: $7.56 \pm 0.71$, RSV-NO: 2.26 \pm 0.67 , VPA-O: $5.27 \pm 0.67$, VPA-NO: 2.35 \pm 0.84 , VPA+RSV-O: $5.37 \pm 0.83$, VPA+RSV-NO: $3.57 \pm 0.99, p<0.001$, Supplementary Figure $3 \mathrm{~A})$.

There were no significant differences in the percentage time (Figure 3C) and distance travelled (Figure 3D) in the door zone and in the inner zone (Supplementary Figure 3C and $3 \mathrm{~B}$, respectively). The animals that did not open the restrainer demonstrated an increased total distance travelled (Con-O: 1810.55 \pm 561.91 , Con-NO: 4280.82 \pm 618.28 , RSV-O: 2777.63 \pm 702.96 , RSV-NO: 4596.10 \pm 1157.95 , VPA-O: 2837.52 \pm 791.57 , VPA-NO: 4329.37 $\pm 1101.45, V P A+R S V-O: 1469.5 \pm 446.61$, VPA+RSV-NO: 5301.62 $\pm 1686.4, p<0.001$, Supplementary Figure 3D). This likely reflects the differences in test length as the test ended after the release of the trapped rat.

\subsection{Parameters of the first day of test correlate with the opener outcome}

Since our data revealed important behavioural differences in the first days of test (Figure $2 \mathrm{C}$ ), we aimed to investigate the possibility of the parameters on the first day of test correlating with the opener outcome. Opener animals were defined as animals that were able to open the restrainer for two consecutive days and to do so at least 3 times during the 12 days of test. We observed a significant increase in the average speed of opener rats, compared to non-openers (Con-OP: 4.50 \pm 1.31 , Con-NP: $4.38 \quad \pm 0.62, \quad$ RSV-OP: 4.66 \pm 1.29 , RSV-NP: $2.77 \pm 0.76$, VPA-OP: $4.19 \pm 0.67$, VPA-NP: $1.89 \pm 0.18$, VPA+RSV-OP: $2.76 \pm 0.32, V P A+R S V-N P: 2.34 \pm 0.51, p=0.021$, Figure $4 A$ ). There is also a significant effect of VPA ( $p=0.002)$, indicating that VPA exposure leads to reduction of the overall speed of both opener and non-opener animals in the first day of test. When taking into account the different regions of the apparatus, our data revealed that opener rats showed increased average speed in the door zone (Con-OP: 4.68 \pm 1.58 , Con-NP: $3.6 \pm 0.35$, RSV-OP: $4.51 \pm 0.56$, RSV-NP: 2.26 \pm 0.35 , VPA-OP: $4.32 \pm 0.68$, VPA-NP: $1.55 \pm 0.22$, VPA+RSV-OP: $2.04 \pm 0.34$, VPA+RSVNP: $1.56 \pm 0.29, p<0.001$, Figure 4B). There is also an effect of VPA and RSV treatments $(p<0.001$ and $p=0.003$, respectively). We also identified differences in average speed in the 
door zone of opener rats from RSV and VPA groups, compared to the VPA+RSV group ( $p<0.001$ and $p=0.017$, respectively) and non-opener rats from the VPA and VPA+RSV groups, compared to the Control group $(p<0.001)$. Openers also showed an elevated average speed in the inner zone (Con-OP: $4.47 \pm 1.44$, Con-NP: $4.44 \pm 0.52$, RSV-OP: $5.08 \pm 1.07$, RSVNP: $2.46 \pm 0.75$, VPA-OP: $4.84 \pm 0.44$, VPA-NP: $1.92 \pm 0.17$, VPA+RSV-OP: $2.80 \pm 0.54$, VPA+RSV-NP: $2.14 \pm 0.38, p<0.001$, Figure $4 C$ ). There is also an effect of VPA treatment, regardless of RSV treatment or opener outcome $(p=0.003)$. We also identified differences in non-opener rats from RSV and VPA when compared to the Control group $(p=0.002, p<0.001$, respectively). Nonetheless, there were no significant differences between groups in the percentages of distance travelled in the door or inner zones (Supplementary Figures 4A and 4B). Interestingly, animals exposed to VPA (regardless of RSV treatment or opener outcome) show a reduction in the distance travelled in the door zone (VPA effect, $p=0.017$, Supplementary Figures $4 \mathrm{~A}$ ), suggesting a possibly reduced investigation of the restrainer door in the first day of test.

The analysis of time spent in the door zone revealed that opener rats from Control group presented an increase in the percentage of time spent in the door zone compared to non-opener rats of the same experimental groups $(p=0.004)$. On the other hand, opener animals of the VPA and VPA+RSV groups showed a reduced percentage of time spent in the door zone compared to non-openers of the same group ( $p=0.029$ and $P=0.005$, respectively, Supplementary Figure 4C). Conversely, there was no significant differences between groups in the time spent in the inner zone (Supplementary Figure 4D) nor total distance travelled (Supplementary Figure 4E).

The previous data indicated important differences between groups that persisted not only on the first day of opening but also on the first day of test (Figure $3 A-B$ and $4 A-C$, respectively). Therefore, we investigated a correlation between parameters of the first day of test and the opener outcome. We found an important positive correlation between opening the restrainer in the first day of test and the opener outcome $(p=0.004)$, with a relative risk (Exp (B)) of 4.83. In addition, our data revealed a significant positive correlation between the average speed and the opener outcome $(p=0.024,(\operatorname{Exp}(B)=1.351)$, with the correlation also maintained in the door zone $(p=0.021,(\operatorname{Exp}(B)=1.545)$, and inner zone $(p=0.022,(\operatorname{Exp}(B)$ $=1.436)$. $($ Table 1$)$ 
Table 1. Correlation between parameters of the first day of test and the opener outcome

\begin{tabular}{l|c|c} 
Parameter of the first day & Relative risk - Exp (B) & p-value \\
\hline Average speed & 1.351 & $0.024^{*}$ \\
Average speed in door zone & 1.545 & $0.021^{*}$ \\
Average speed in inner zone & 1.436 & $0.022^{*}$ \\
Distance travelled & 1 & 0.866 \\
Percentage distance in inner zone & 1.050 & 0.419 \\
Percentage distance in door zone & 1.015 & 0.834 \\
Percentage time in inner zone & 1.021 & 0.327 \\
Percentage time in door zone & 0.991 & 0.757 \\
Releasing trapped animal & 4.83 & 0.004 **
\end{tabular}

Average speed and releasing the trapped animal on the first day of test are positively correlated with the opener outcome. ${ }^{*} p<0.05 ;{ }^{* *} p<0.01$ 


\section{DISCUSSION}

The ability to understand another's feelings and perceptions, and the desire to improve the well-being of others, is crucial for successful social interactions and the survival of social groups [12]. Thus, empathy is an integral part of social behaviour. Individuals within the autism spectrum show impairments in social interaction and communication and are usually thought to present an altered experience or expression of empathy. Cognitive empathy (the ability to understand what others are feeling or thinking) appears to be primarily impaired in those with ASD, while affective empathy (the capacity to share other people's feelings while understanding they are different from one's own) is either preserved [14, 21, 27] or impaired only when emotions of negative valence are involved [32].

Although empathy is a complex behaviour in humans, simplified models could be useful to investigate the behavioural and neural processes that constitute the primal emotional foundation of empathy [34]. In the past years, rodents have been recognized as a reliable model to study empathy-like pro-social behaviour and new experimental protocols have been developed $[23,39]$. In the present work, we proposed to use one of these new methods to evaluate pro-social behaviour in the animal model of autism induced by VPA. We showed that VPA animals are able to open a restrainer to release a trapped conspecific, and continue to do so at the same frequency as controls once they learn this behaviour. This suggests that VPA rats also present pro-social behaviour and take action to alleviate the suffering of another animal in distress.

Interestingly, rats of the VPA and VPA+RSV groups showed a delay in the expression of this helping behaviour, opening the restrainer for the first time on average 3 days later than Controls. Thus, it is possible that VPA animals are less able to express an adequate response to the distress of the trapped animal. This is supported by a study using the BTBR T (+) Itpr3(tf)/J mouse model of autism, in which mice successfully recognize stress in a conspecific, but fail to express the typical response (increased social interaction with the stressed mouse) [33]. An intriguing hypothesis is that the emotional response of animal models of autism is abnormal. Indeed, both VPA rats and BTRB mice show impaired emotional learning in a fear conditioning task [4, 31], indicating alterations in their neural processing of emotions. An alternative explanation for the delay of VPA animals to open the restrainer is that they have an impaired communication and, as a consequence, show difficulties to recognize that the trapped animal is in distress. In that case, it is likely that ultrasonic vocalizations emitted by the rat in the restrainer would be essential to communicate its distress [25]. It is possible that VPA animals do not recognize this call, since they present an aberrant pattern of ultrasonic vocalization in situations of stress [26] and an altered auditory processing of these vocalizations [17]. Animals exposed to VPA could also have decreased attention towards the 
restrainer or reduced motivation to interact with it. Our data shows no differences between groups in the time spent near the door or other regions of the apparatus, indicating that exploration of the apparatus seems to be similar between groups. However, our behavioural paradigm does not allow for the direct assessment of attention or motivation.

Anxiety is a major comorbidity in ASD with a prevalence in individuals with autism that varies from $42 \%$ to $79 \%$, which is significantly higher than the general population [22]. Anxiety is also widely identified in animal models of autism, including the VPA model [15]. Therefore, anxious behaviour of rats exposed to VPA could hamper the initial interaction with the restrainer and result in a delayed first opening. In fact, animals exposed to VPA showed a significant reduction in the distance travelled in the door zone in the first day of test (Supplementary Figure $4 \mathrm{~A}$ ), which could reflect an effect of different levels of anxiety. Our behavioural paradigm does not allow for the direct evaluation of anxiety during the test. Studying the impact of anxiety on the expression of pro-social behaviour could be a focus of future research.

Empathy-like behaviours in animals can be a very complex social phenomenon. The degree of affiliation between rats likely modulates this behaviour, and may involve complex cognitive processes [11,34]. Rats have a natural tendency (not conditioned) to express empathy-like behaviours [6, 45]. In humans, observation of other people's states activates many of the same motor, sensory and limbic brain regions involved in experiencing this state in of itself [24, 35]. Studies have shown that the anterior cingulate cortex (ACC), medial orbitofrontal cortex (mOFC) and medial prefrontal cortex (mPFC), might be related to social behaviours associated with the perception of others' feelings and empathy in both rats and humans [36]. In order to express pro-social behaviours, animals and humans also likely need to combine information of different sensory modalities over time [11, 40]. The VPA model shows deficits in somatosensory processing that are prevented by RSV [16]. Therefore, it is unlikely that the delayed expression of empathy-like behaviour in VPA animals is caused by impaired primary sensorial processing. However, it is possible that integration of the sensorial information in other cognitive process could be affected. Indeed, there are many reports suggesting impairments in decision making processes in ASD [29, 42] due, at least in part, to a reduced tendency to incorporate emotional information [47]. Therefore, the deficits seen in VPA animals in the present work might be reflecting alterations in cognitive processes such as the integration of sensory information in the decision making process and the interpretation of emotional states.

In our analyses, we also investigated which parameters were associated with the act of opening the restrainer. We found that on the day of opening, animals that opened moved 
faster compared to littermates that did not open the apparatus. In the first day of test, this increased speed was also present in the group of animals that later became openers when compared to non-openers. Thus, it seems that animal that actively investigate the restrainer, moving faster during the duration of the test, have a higher propensity to open the apparatus. Specifically, a generalized linear model detected a significant association between the opener outcome and average speed, average speed in the door zone and average speed in the inner zone in the first day of test (35\%,54\% and 44\%-increased odds, respectively). In the same analysis, we found that animals that opened the apparatus on the first day of test had a higher chance of becoming an opener (383\%-increased odds). This result suggests that learning to release the trapped animal early, specifically on the first day of test, impacts the later manifestation of this behaviour. However, whether this result is a cause or consequence of this behaviour is not clarified by our approach.

Finally, prenatal RSV treatment showed no significant effect with regards to preventing the deficits of empathy-like behaviour in VPA animals. Since RSV can prevent social impairments in the VPA model of autism $[3,20]$, the pro-social behaviour evaluated in our paradigm could require a different neural substrate, indicating that social and empathy-like behaviours are distinct from one another. According to our results, it is also possible that VPA does not directly affect circuits related to empathy, but modulates processes such as decisionmaking or anxiety, which could not be affect by RSV. We also cannot rule out that dose and treatment regimen of RSV are not enough to show any beneficial effect.

\section{CONCLUSIONS}

In conclusion, we investigated empathy-like pro-social behaviour in an animal model of autism for the first time. We showed that VPA hinders the timely manifestation of empathy-like behaviour, but does not affect the continuation of this behaviour after its first expression. Moreover, learning to release a conspecific from the restrainer seemed to be facilitated by being able to open the apparatus in the first day of test and by higher speeds in this same day. Further investigation of empathy-like behaviour in the VPA model and in other models of autism could help to clarify the behavioural and neural processes underpinning the basic aspects of empathy alterations in autistic individuals. 


\section{FUNDING AND DISCLOSURE}

This work was supported by the National Institute of Science and Technology on Neuroimmunomodulation-INCT-NIM \#465489/2014-1; National Council of Technological and Scientific Development (CNPq); Coordination for the Improvement of Higher Education Personnel (CAPES), Clinical Hospital of Porto Alegre (FIPE-HCPA), PROPESQ-UFRGS.

Prof Gottfried and Dr Bambini-Junior would like to disclose they are inventors of a patent related to the methodology used in this work, however they do not own it (it is deposited and owned by the Universidade Federal do Rio Grande do Sul), and would not have any financial benefit from it. All other authors declare no potential conflict of interest.

\section{ACKNOWLEDGMENTS}

We would like to thank the statistical support group from the HCPA, the engineering group from HCPA for the technical drawings for building the restrainers, Fluxome (Stenløse, Denmark) for the generous gift of resveratrol, and the online infographic maker, Mind the Graph (attribution share-alike 4.0 licensing) for the templates of schematic figures. 


\section{REFERENCES}

[1] APA, Diagnostic and Statistical Manual of Mental Disorders. American Psychiatric Association, 2013.

[2] V. Bambini-Junior, L. Rodrigues, G.A. Behr, J.C. Moreira, R. Riesgo, C. Gottfried, Animal model of autism induced by prenatal exposure to valproate: behavioral changes and liver parameters, Brain research 1408 (2011) 8-16.

[3] V. Bambini-Junior, G. Zanatta, G. Della Flora Nunes, G. Mueller de Melo, M. Michels, M. Fontes-Dutra, V. Nogueira Freire, R. Riesgo, C. Gottfried, Resveratrol prevents social deficits in animal model of autism induced by valproic acid, Neuroscience letters 583 (2014) 176-181.

[4] A. Banerjee, C.T. Engineer, B.L. Sauls, A.A. Morales, M.P. Kilgard, J.E. Ploski, Abnormal emotional learning in a rat model of autism exposed to valproic acid in utero, Frontiers in behavioral neuroscience 8 (2014) 387.

[5] S. Baron-Cohen, S. Wheelwright, The empathy quotient: an investigation of adults with Asperger syndrome or high functioning autism, and normal sex differences, Journal of autism and developmental disorders 34 (2004) 163-175.

[6] I. Ben-Ami Bartal, J. Decety, P. Mason, Empathy and pro-social behavior in rats, Science (New York, N.Y.) 334 (2011) 1427-1430.

[7] I. Ben-Ami Bartal, D.A. Rodgers, M.S. Bernardez Sarria, J. Decety, P. Mason, Prosocial behavior in rats is modulated by social experience, eLife 3 (2014) e01385.

[8] C.T. Chiu, Z. Wang, J.G. Hunsberger, D.M. Chuang, Therapeutic potential of mood stabilizers lithium and valproic acid: beyond bipolar disorder, Pharmacological reviews 65 (2013) 105-142.

[9] J. Christensen, T.K. Gronborg, M.J. Sorensen, D. Schendel, E.T. Parner, L.H. Pedersen, M. Vestergaard, Prenatal valproate exposure and risk of autism spectrum disorders and childhood autism, Jama 309 (2013) 1696-1703.

[10] R.M. Church, Emotional reactions of rats to the pain of others, Journal of comparative and physiological psychology 52 (1959) 132-134.

[11] F.B. de Waal, Putting the altruism back into altruism: the evolution of empathy, Annu Rev Psychol 59 (2008) 279-300.

[12] J. Decety, M. Svetlova, Putting together phylogenetic and ontogenetic perspectives on empathy, Developmental cognitive neuroscience 2 (2012) 1-24.

[13] U. Dimberg, M. Thunberg, Empathy, emotional contagion, and rapid facial reactions to angry and happy facial expressions, PsyCh journal 1 (2012) 118-127.

[14] I. Dziobek, K. Rogers, S. Fleck, M. Bahnemann, H.R. Heekeren, O.T. Wolf, A. Convit, Dissociation of cognitive and emotional empathy in adults with Asperger syndrome using the Multifaceted Empathy Test (MET), Journal of autism and developmental disorders 38 (2008) 464-473.

[15] M.R. Favre, T.R. Barkat, D. Lamendola, G. Khazen, H. Markram, K. Markram, General developmental health in the VPA-rat model of autism, Frontiers in behavioral neuroscience 7 (2013) 88.

[16] M. Fontes-Dutra, J. Santos-Terra, I. Deckmann, G. Brum Schwingel, G. Della-Flora Nunes, M.M. Hirsch, G. Bauer-Negrini, R.S. Riesgo, V. Bambini-Junior, C. HedinPereira, C. Gottfried, Resveratrol Prevents Cellular and Behavioral Sensory Alterations in the Animal Model of Autism Induced by Valproic Acid, Front Synaptic Neurosci 10 (2018) 9.

[17] M.J. Gandal, J.C. Edgar, R.S. Ehrlichman, M. Mehta, T.P. Roberts, S.J. Siegel, Validating gamma oscillations and delayed auditory responses as translational biomarkers of autism, Biological psychiatry 68 (2010) 1100-1106.

[18] D.H. Geschwind, Advances in autism, Annual review of medicine 60 (2009) 367-380. 
[19] C. Gottfried, V. Bambini-Junior, F. Francis, R. Riesgo, W. Savino, The Impact of Neuroimmune Alterations in Autism Spectrum Disorder, Frontiers in psychiatry 6 (2015) 121.

[20] M.M. Hirsch, I. Deckmann, M. Fontes-Dutra, G. Bauer-Negrini, G. Della-Flora Nunes, W. Nunes, B. Rabelo, R. Riesgo, R. Margis, V. Bambini-Junior, C. Gottfried, Behavioral alterations in autism model induced by valproic acid and translational analysis of circulating microRNA, Food and chemical toxicology : an international journal published for the British Industrial Biological Research Association 115 (2018) 336343.

[21] A.P. Jones, F.G. Happe, F. Gilbert, S. Burnett, E. Viding, Feeling, caring, knowing: different types of empathy deficit in boys with psychopathic tendencies and autism spectrum disorder, Journal of child psychology and psychiatry, and allied disciplines 51 (2010) 1188-1197.

[22] R. Kent, E. Simonoff, Prevalence of Anxiety in Autism Spectrum Disorders, 2017.

[23] S. Keum, H.S. Shin, Rodent models for studying empathy, Neurobiology of learning and memory 135 (2016) 22-26.

[24] C. Keysers, J.H. Kaas, V. Gazzola, Somatosensation in social perception, Nat Rev Neurosci 11 (2010) 417-428.

[25] E.J. Kim, E.S. Kim, E. Covey, J.J. Kim, Social transmission of fear in rats: the role of 22-kHz ultrasonic distress vocalization, PloS one 5 (2010) e15077.

[26] H.Y. Kuo, F.C. Liu, Valproic acid induces aberrant development of striatal compartments and corticostriatal pathways in a mouse model of autism spectrum disorder, FASEB journal : official publication of the Federation of American Societies for Experimental Biology 31 (2017) 4458-4471.

[27] P.L. Lockwood, G. Bird, M. Bridge, E. Viding, Dissecting empathy: high levels of psychopathic and autistic traits are characterized by difficulties in different social information processing domains, Frontiers in human neuroscience 7 (2013) 760.

[28] W. Loscher, Basic pharmacology of valproate: a review after 35 years of clinical use for the treatment of epilepsy, CNS drugs 16 (2002) 669-694.

[29] L. Luke, I.C. Clare, H. Ring, M. Redley, P. Watson, Decision-making difficulties experienced by adults with autism spectrum conditions, Autism 16 (2012) 612-621.

[30] D.F. Mabunga, E.L. Gonzales, J.W. Kim, K.C. Kim, C.Y. Shin, Exploring the Validity of Valproic Acid Animal Model of Autism, Experimental neurobiology 24 (2015) 285-300.

[31] P. MacPherson, R. McGaffigan, D. Wahlsten, P.V. Nguyen, Impaired fear memory, altered object memory and modified hippocampal synaptic plasticity in split-brain mice, Brain research 1210 (2008) 179-188.

[32] M. Mazza, M.C. Pino, M. Mariano, D. Tempesta, M. Ferrara, D. De Berardis, F. Masedu, M. Valenti, Affective and cognitive empathy in adolescents with autism spectrum disorder, Frontiers in human neuroscience 8 (2014) 791.

[33] K. Meyza, T. Nikolaev, K. Kondrakiewicz, D.C. Blanchard, R.J. Blanchard, E. Knapska, Neuronal correlates of asocial behavior in a BTBR T (+) Itpr3(tf)/J mouse model of autism, Frontiers in behavioral neuroscience 9 (2015) 199.

[34] K.Z. Meyza, I.B. Bartal, M.H. Monfils, J.B. Panksepp, E. Knapska, The roots of empathy: Through the lens of rodent models, Neuroscience and biobehavioral reviews 76 (2017) 216-234.

[35] P. Molenberghs, R. Cunnington, J.B. Mattingley, Brain regions with mirror properties: a meta-analysis of 125 human fMRI studies, Neurosci Biobehav Rev 36 (2012) 341 349.

[36] T.J. Mony, M. Hong, H.J. Lee, Empathy Study in Rodent Model of Autism Spectrum Disorders, Psychiatry Investig 15 (2018) 104-110.

[37] S.J. Moore, P. Turnpenny, A. Quinn, S. Glover, D.J. Lloyd, T. Montgomery, J.C. Dean, A clinical study of 57 children with fetal anticonvulsant syndromes, Journal of medical genetics 37 (2000) 489-497.

[38] C. Onore, M. Careaga, P. Ashwood, The role of immune dysfunction in the pathophysiology of autism, Brain, behavior, and immunity 26 (2012) 383-392. 
[39] J. Panksepp, J.B. Panksepp, Toward a cross-species understanding of empathy, Trends in neurosciences 36 (2013) 489-496.

[40] D. Raposo, J.P. Sheppard, P.R. Schrater, A.K. Churchland, Multisensory decisionmaking in rats and humans, J Neurosci 32 (2012) 3726-3735.

[41] G.E. Rice, P. Gainer, "Altruism" in the albino rat, Journal of comparative and physiological psychology 55 (1962) 123-125.

[42] S. Robic, S. Sonie, P. Fonlupt, M.A. Henaff, N. Touil, G. Coricelli, J. Mattout, C. Schmitz, Decision-making in a changing world: a study in autism spectrum disorders, J Autism Dev Disord 45 (2015) 1603-1613.

[43] P.M. Rodier, J.L. Ingram, B. Tisdale, V.J. Croog, Linking etiologies in humans and animal models: studies of autism, Reproductive toxicology (Elmsford, N.Y.) 11 (1997) 417-422.

[44] K.M. Rump, J.L. Giovannelli, N.J. Minshew, M.S. Strauss, The development of emotion recognition in individuals with autism, Child development 80 (2009) 1434-1447.

[45] N. Sato, L. Tan, K. Tate, M. Okada, Rats demonstrate helping behavior toward a soaked conspecific, Animal cognition 18 (2015) 1039-1047.

[46] T. Schneider, R. Przewlocki, Behavioral alterations in rats prenatally exposed to valproic acid: animal model of autism, Neuropsychopharmacology : official publication of the American College of Neuropsychopharmacology 30 (2005) 80-89.

[47] P. Shah, C. Catmur, G. Bird, Emotional decision-making in autism spectrum disorder: the roles of interoception and alexithymia, Mol Autism 7 (2016) 43.

[48] R. Tyzio, R. Nardou, D.C. Ferrari, T. Tsintsadze, A. Shahrokhi, S. Eftekhari, I. Khalilov, V. Tsintsadze, C. Brouchoud, G. Chazal, E. Lemonnier, N. Lozovaya, N. Burnashev, Y. Ben-Ari, Oxytocin-mediated GABA inhibition during delivery attenuates autism pathogenesis in rodent offspring, Science (New York, N.Y.) 343 (2014) 675-679.

[49] H. Walter, Social Cognitive Neuroscience of Empathy: Concepts, Circuits, and Genes:, Emotion Review 4 (2012). 


\section{c. FIGURE LEGENDS (Figures are embedded for ease of reading)}

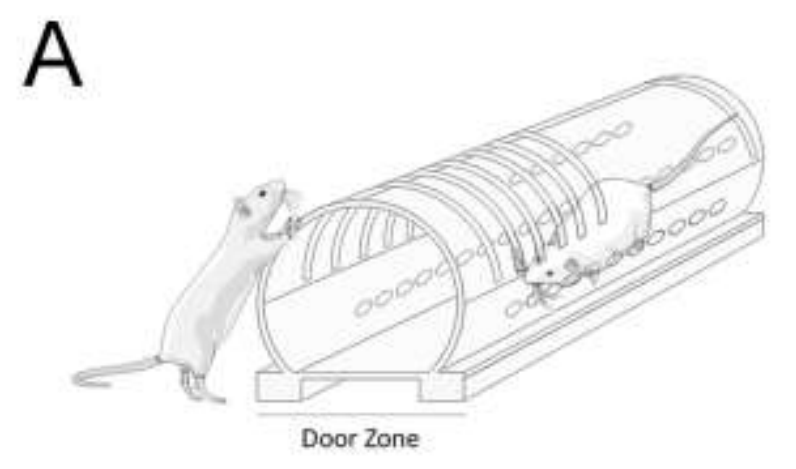

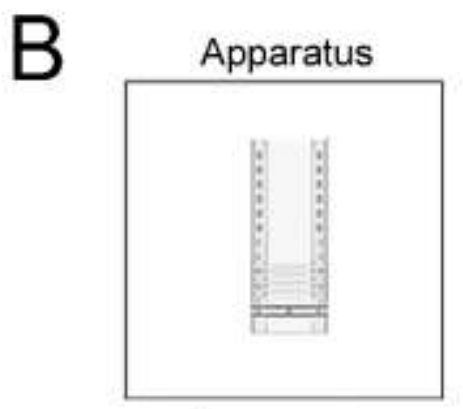

Inner zone

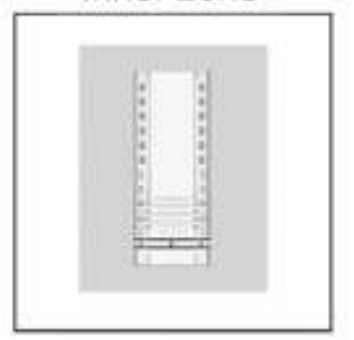

Outer zone

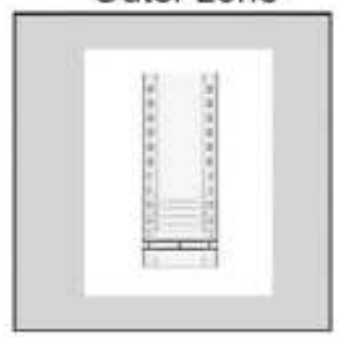

Door zone

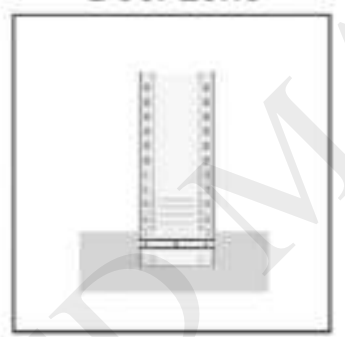

Figure 1. Schematic representation of the test apparatus $(\mathbf{A})$ and the regions considered in the analyses (B). The inner zone was determined so that its boundaries are at half the distance between the edge of the apparatus and the wooden box. The door zone is the part of the inner zone surrounding the front of the apparatus. 


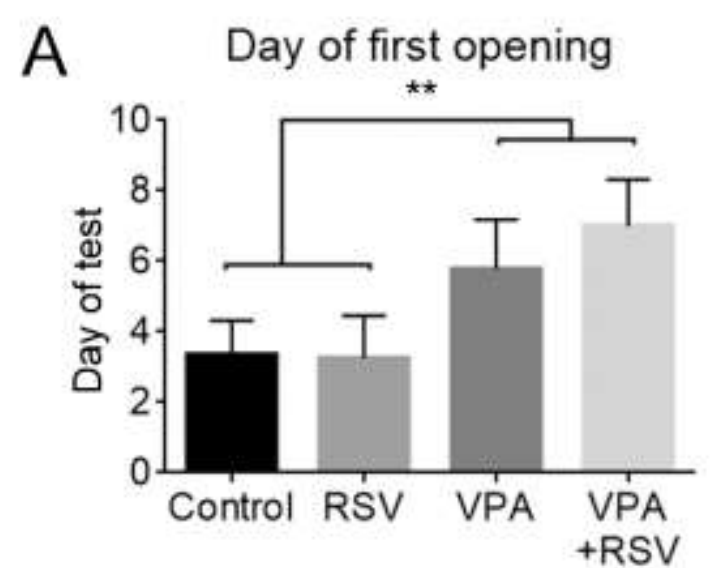

C

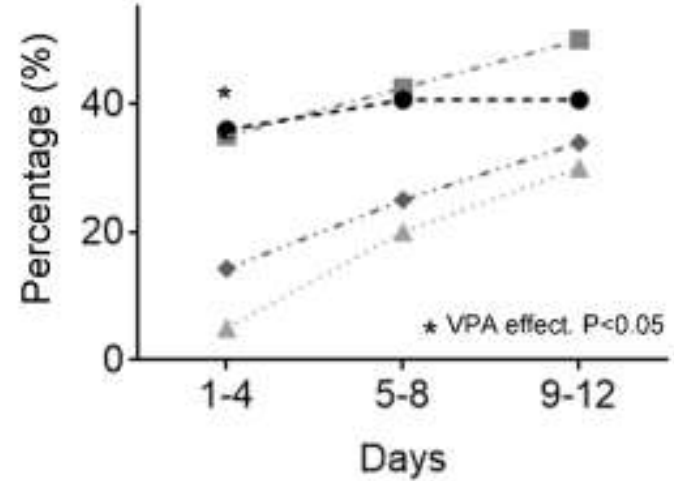

- Control - $=$ RSV - VPA A VPA+RSV
B

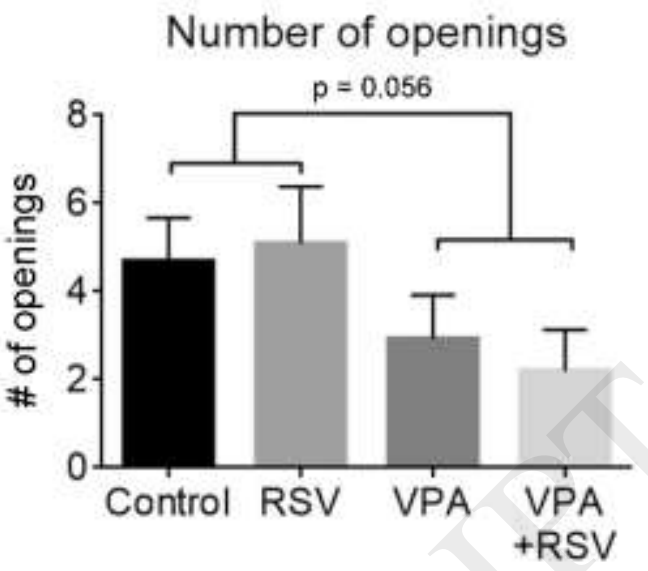

D

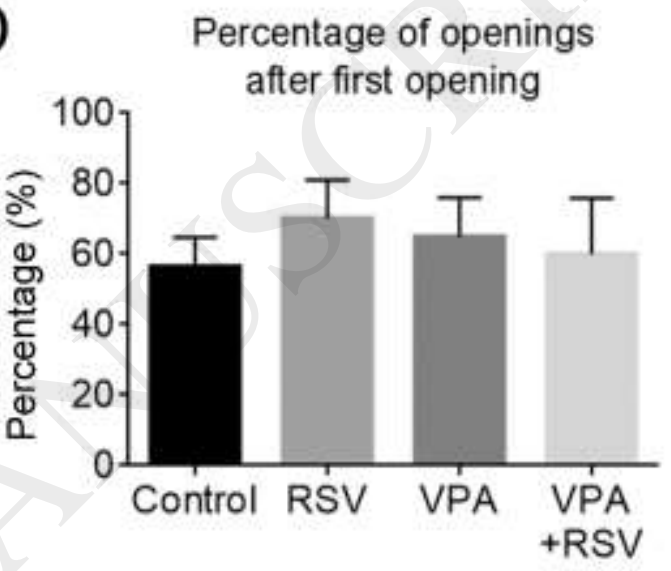

Figure 2. Prenatal exposure to VPA hinders the timely manifestation of empathy-like behaviour. The day of first opening is delayed $(\mathbf{A})$ and total number of openings shows a tendency towards reduction (B) in animals prenatally exposed to VPA (regardless of treatment with RSV). VPA leads to a diminished percentage of openings on days 1 to 4 of test, but does not affect the percentage number of openings in the later days of test (C) or the percentage of openings after the day of first opening (D). Control $n=16, R S V n=10$, VPA $n=14$, VPA + RSV $n=10$. In “D”, Control $n=14$, RSV $n=8$, VPA $n=9$, VPA + RSV $n$ $=7 .{ }^{*} p<0.05,{ }^{* *} p<0.01$. 


\section{Average Speed}

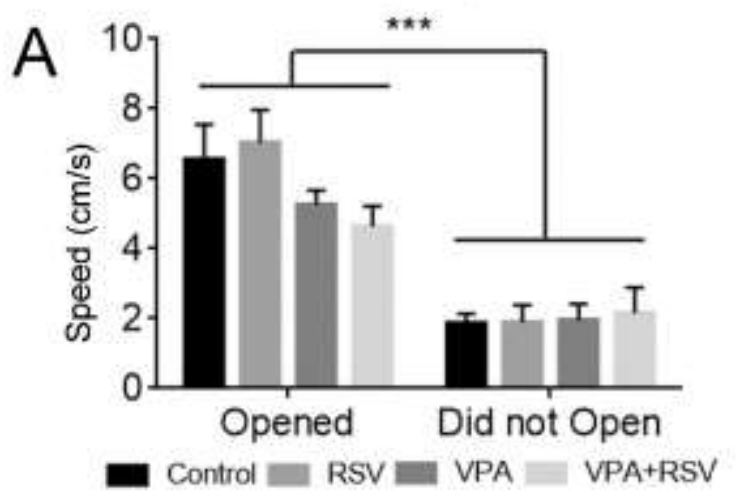

C Percentage time in door zone

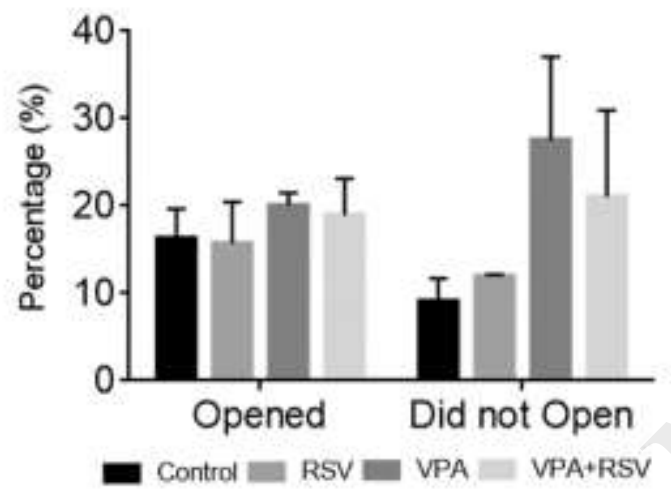

Average Speed in Door Zone

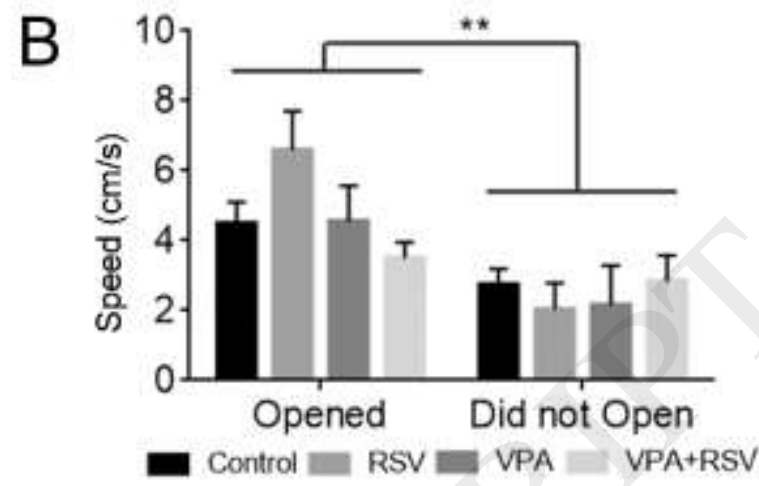

D

Percentage distance in door zone

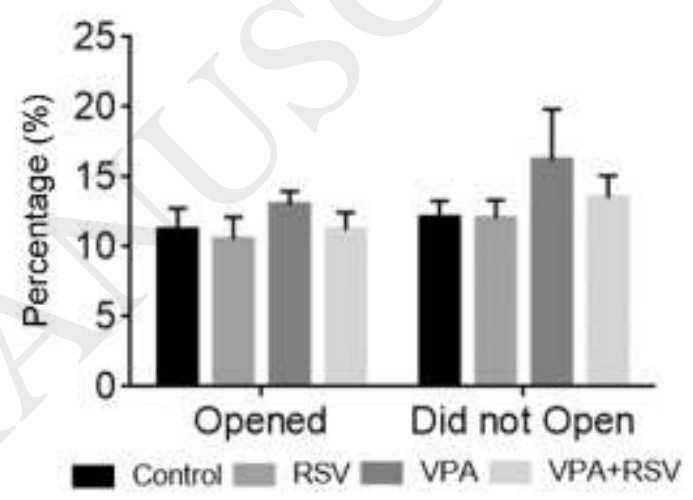

Figure 3. The speed of animals during the day of first opening is higher compared to littermates that were unable to open the apparatus. We evaluated the behaviour of 2 animals of the same litter on the day of first opening of one of them. Animals were excluded from this analysis if both animals opened the apparatus on the same day. On the day of first opening, rats moved faster in the entire apparatus $(\mathbf{A})$ and in the door zone $(\mathbf{B})$ compared to littermates that did not open the apparatus on that day (regardless of treatment). However, there are no differences in the percentage of time spent in the door zone $(\mathbf{C})$ or percentage of distance travelled in the door zone (D). Opened: Control $n=7, \operatorname{RSV} n=3, \operatorname{VPA} n=4$, $\mathrm{VPA}+\mathrm{RSV} \mathrm{n}=4$. Did not open: Control $\mathrm{n}=7, \mathrm{RSV} \mathrm{n}=3, \mathrm{VPA} \mathrm{n}=4, \operatorname{VPA}+\operatorname{RSV} \mathrm{n}=4^{* \star} p$ $<0.01,{ }^{* * *} p<0.001$. 

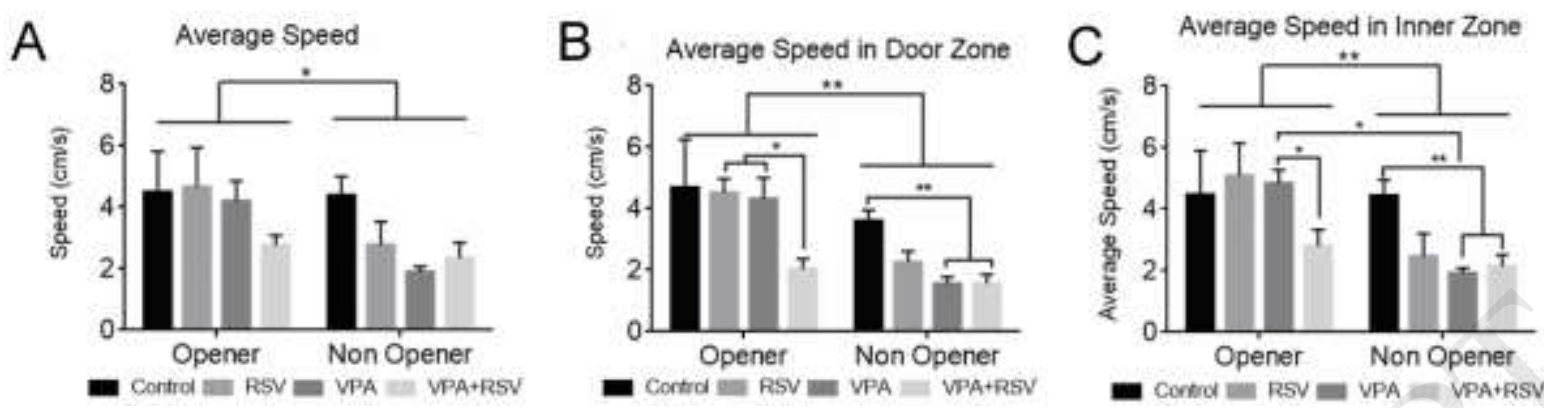

Figure 4. Opener animals move faster than non-openers on the first day of test. Opener and non-openers present a significant difference in the average speed $(\mathbf{A})$ speed in the door zone (B) and speed in the Inner zone (C) on the first day of test. There is also a significant effect of VPA treatment, causing reduced average speed (regardless of RSV treatment or opener outcome). VPA effect: $p<0.01$ in $\mathbf{A}$ and $\mathbf{C}$ and $p<0.001$ in $\mathbf{B}$. Openers: Control $n=7$, $R S V n=4$, VPA $n=4$, VPA + RSV $n=2$. Non-openers: Control $n=9, R S V n=6$, VPA $n=$ $10, \mathrm{VPA}+\mathrm{RSV} n=8{ }^{*} p<0.05,{ }^{* *} p<0.01$. 